Bol. Acad. peru. leng. 65. 2019 (87-100)

\title{
EL CONCEPTO DE PLAGIO DESDE LA PERSPECTIVA DE UN GRUPO DE ESTUDIANTES UNIVERSITARIOS PERUANOS
}

\section{THE CONCEPT OF PLAGIARISM FROM THE PERSPECTIVE OF A GROUP OF PERUVIAN UNIVERSITY STUDENTS}

\author{
Teresa Ramos Quispe \\ Universidad Nacional de San Agustín de Arequipa \\ Dennis Arias Chávez \\ Universidad Continental, Arequipa
}

\section{Resumen:}

El plagio, considerado como acto deshonesto, ha sido denunciado tanto por instituciones académicas como por no académicas. Dada la gravedad y repercusión del hecho, sus implicaciones en la actualidad van más allá de las sanciones reglamentarias y su alcance llega incluso a lo legal y penal. En el presente estudio, se analiza un grupo de definiciones producto de la información proporcionada por un grupo de 200 estudiantes de una universidad privada peruana con el fin de identificar cuáles son las palabras con las que califican este acto, cuáles son los productos académicos susceptibles de plagiar y cuál es la definición que se desprende de lo manifestado por los participantes. El estudio es de corte cualitativo. A cada participante se le entregó una ficha codificada con tres preguntas sobre datos generales (edad, sexo y carrera), y una pregunta de tipo 
abierta en la que se les solicita definir en sus palabras el término plagio. Los resultados indican que los términos con mayor presencia en las definiciones son copia, persona, examen, información y trabajos (académicos). En esta investigación, finalmente, se propone una definición de plagio sobre la base de lo expresado por los participantes.

\section{Abstract:}

Plagiarism, considered as a dishonest act, has been denounced by both academic and non-academic institutions. Given the seriousness and impact of the event, its implications nowadays go beyond regulatory sanctions and its scope even reaches legal and criminal matters. In the present study, a group of definitions is analyzed, product of the information provided by a group of 200 students of a Peruvian private university in order to identify which are the words with which they qualify this act, which are the academic products susceptible of plagiarize and what is the definition that emerges from what is stated by the participants. The study is qualitative. Each participant was given a coded record with three questions on general data (age, gender and career) and an open-ended question in which they are asked to define in their words the term plagiarism. The results indicate that the terms with the greatest presence in the definitions are copy, person, exam, information and works (academic). In this investigation, finally, a definition of plagiarism is proposed based on what was expressed by the participants.

Palabras clave: plagio, copia, producto académico, universidad, implicancia.

Key words: plagiarism, copy, academic product, university, implication.

Fecha de recepción:

$15 / 03 / 2019$

Fecha de aceptación:

$31 / 05 / 2019$ 
https://doi.org/10.46744/bapl.201901.005

\section{Introducción}

Los estudios sobre el fenómeno del plagio son de reciente data. No es sino en los años noventa cuando surge el interés por la deshonestidad académica con el fin de combatir el fenómeno del plagio en las instituciones educativas. Algunos estudios resultan pioneros en este campo, entre ellos destacan los realizados por McCabe y Trevino (1993), Hexman (1999), Jordan (2001) o Lambert, Hogan y Barton (2003), quienes centran su interés en estudiar las repercusiones del plagio en las aulas de los campus norteamericanos. Este interés no resulta gratuito, sino que es producto del avance de la tecnología y de su impacto en la vida de las personas. La aparición de la internet, así como el uso masivo de ordenadores no solo en las universidades sino también en el hogar y el trabajo facilitó la aparición de nuevas formas de copiar información con el fin de hacerla pasar como propia, fenómeno que los especialistas han denominado ciberplagio (Sureda, Comas y Morey, 2009).

La accesibilidad a estas nuevas formas de comunicación ha facilitado la aparición e incremento de casos de plagio, no solo en el ámbito académico, en el que suele ser una práctica constante, sino también en otros campos como el artístico y político. No debe sorprender, por ello, que en los últimos años el interés por estudiar este fenómeno haya crecido. En 2011, por ejemplo, Molina, Velásquez, Ríos, Calfucoy y Cociña determinaron que más del $50 \%$ de estudiantes chilenos de educación secundaria y el $40 \%$ de educación superior admitieron haber tomado información de internet sin citar la fuente. A esto se suma lo hallado por Sureda et al., quienes, en 2009, realizaron un estudio en una universidad mexicana con estudiantes de 15 años en adelante, el cual dio como resultado que más del $44 \%$ de estudiantes habían cometido ciberplagio (información tomada de internet sin citar), en tanto que el $30 \%$ admitió haber «copiado y pegado» información de internet en sus trabajos académicos.

Los estudios antes señalados se complementan con aquellos relacionados con la escritura deshonesta (Espinosa, Castellarin, y Biagioni, 
2013), así como también con los que centran su interés en conocer la percepción sobre el plagio académico en estudiantes universitarios (Castro, Yoplac-López, Carpio-Tello, Sihuay-Torres \& Cósar-Quiroz, 2017; Cebrián-Robles, Raposo, Rivas, Cebrián de la Serna y SarmientoCampos, 2018), los estudios sobre las atribuciones causales en el plagio académico por parte de los estudiantes universitarios (Rebollo-Quintela, Espiñeira-Bellón y Muñoz-Cantero, 2017), las representaciones sociales que tiene el sector sobre el plagio en la escritura académica (López-Gil y Fernández-López 2019) y las actitudes que presentan los estudiantes universitarios hacia este acto (Ramos et al., 2018).

En vista de la necesidad de estudiar aspectos esenciales como el concepto que se tiene de este acto, el presente estudio se centra en identificar y explicar cuáles son las palabras con las que los estudiantes universitarios califican el plagio; así también, determinar cuáles son los productos académicos susceptibles de plagiar y cuál es la definición que se desprende de lo manifestado por los participantes. Para estudiar analizar todo ello, esta investigación se ordena de la siguiente manera: se presenta las definiciones sobre plagio en las fuentes académicas, se describe la metodología, se exponen los resultados, en el que se da respuesta a nuestras tres preguntas de indagación y, finalmente, se plantean las conclusiones.

\section{Definiciones de plagio}

En su sentido tradicional, el Diccionario de la lengua española (DLE), de la Real Academia Española (RAE) define la acción de plagiar como 'copiar en lo sustancial obras ajenas, dándolas como propias', definición que coincide con la del Diccionario Panbispánico de Dudas. En este sentido, plagiar implica hacerse de la obra de un tercero para beneficio propio. En el campo del Derecho, el plagio se puede concebir desde el ámbito del Derecho Intelectual, en el que plagiar se relaciona con el arte y la literatura, ya que las obras artísticas son susceptibles de ser copiadas, imitadas o robadas con el propósito de obtener beneficios o engañar. En esta misma línea, el glosario de la Organización Mundial de la Propiedad Intelectual (citado en Rojas, 2016) define plagio como el 
https://doi.org/10.46744/bapl.201901.005

acto de ofrecer o presentar como propia, en su totalidad o en parte, la obra de otra persona, en una forma o contexto más o menos alterados. Similar concepción mantiene, para el contexto peruano, el Registro Nacional de Trabajos de Investigación (RENATI, 2016), institución que define como plagio al apoderamiento de todos o de algunos elementos originales contenidos en la obra de otro autor y que son presentados como propios, ya sea haciendo pasar la obra como propia o bien utilizando los elementos creativos de aquella para la elaboración de la obra ilegítima (sunedu: Resolución del Consejo Directivo, 2016, p. 598705). Estas dos últimas acepciones, a diferencia del DLE, amplían el panorama para el entendimiento de este fenómeno al agregar en la definición el factor «intencionalidad o premeditación para realizar el acto».

Por otra parte, algunos autores han ampliado el alcance semántico de este término, llevándolo al campo de la producción intelectual. Por ejemplo, Rosselot et al. (2008) agregan dos factores importantes a la definición tradicional de plagio: «reconocimiento de las ideas» y «acto fraudulento». Para dichos autores:

Plagio es la apropiación, presentación y utilización de material intelectual ajeno, sin el debido reconocimiento de su fuente original. Constituye, por lo tanto, un acto fraudulento, en el cual existe presunción de intencionalidad, en el sentido de hacer parecer un determinado conocimiento, labor o trabajo, como producto propio; y de desconocer la participación de otros en su generación, aplicación o en su perfeccionamiento (p. 136).

Girón (2008), por su parte, incide en que en todo acto de plagio existe intencionalidad de hacerlo Para el autor, quien copia las ideas sin dar el crédito correspondiente comete una suerte de robo intelectual. Así mismo, en el campo académico esta apropiación de información en beneficio propio constituye fraude que las instituciones superiores sancionan con penas que van desde llamadas de atención hasta el retiro de los grados y títulos obtenidos. Para Girón, existe plagio cuando se presentan los siguientes casos: 
Se toman ideas o palabras escritas por otros sin reconocer de forma directa el haberlo hecho $[\ldots]$. Se produce también al presentar como propio un trabajo de forma parcial o total sin ser el autor o autora de dicho trabajo [...]. Al actuar de mala fe deliberadamente al copiar la propiedad intelectual de otros para producir un daño a los autores originales.

[...]. Se considera que se comete plagio al copiar cualquier objeto de fondo o de forma, ya sea una situación, un desarrollo o incluso una simple frase (p. 6).

Entre los objetivos de la universidad se encuentra el de formar profesionales que se desenvuelvan con solvencia en su campo. Para ello, los estudiantes necesitan aprender de las materias que se les imparte, así como de la experiencia de los docentes. La producción de textos académicos se mueve dentro de contextos en los que prima un intercambio definido por instituciones formales, esto es, que a diferencia de otros contextos en los que existe mayor libertad de creación, el ámbito académico espera que todo aquel que quiera generar textos que sean aceptados conozca y respete adecuadamente las convenciones comunicativas y lingüísticas propias de esta práctica (Ibáñez, Tagliabure y Zangaro, 2007). Para lograrlo, el estudiante debe hacerse cuestionamientos y reformular sus significados mediante procesos como la deducción e inducción, y contrastando sus ideas; solo así el discurso que generen será sólido y con una fuerza argumentativa efectiva (Lopera, Ramírez, Zuluaga y Ortíz, 2010). No obstante, en muchos estudiantes que inician su experiencia universitaria aún persisten hábitos traídos de niveles anteriores como la secundaria, en la que el aprendizaje se forja básicamente sobre el discurso del profesor y los libros de texto. La universidad exige que los estudiantes establezcan diálogos con distintos autores con la intención de sentar las bases de un discurso propio. Pero si este diálogo se convierte simplemente en una repetición interminable de citas sin análisis, no habrá una construcción adecuada del conocimiento (Bereiter y Scardamalia, 1992). Esta es una de las razones que motivaron la realización del presente estudio: saber qué entienden los estudiantes por plagio y cuáles son sus implicancias, ya que ello ayudará a predecir y corregir futuros casos de fraude y mejorar el aspecto formativo y la calidad de la información usada en el proceso formativo de la juventud estudiosa 
https://doi.org/10.46744/bapl.201901.005

\section{Metodología}

La presente investigación es de corte cualitativo. Los datos se obtuvieron a partir de la información proporcionada por un grupo de 200 estudiantes del primer semestre de ocho carreras profesionales de una universidad privada en Arequipa. Previamente al desarrollo del estudio, se realizaron las coordinaciones tanto con los responsables de carrera, así como con los profesores de los ocho grupos del curso general de Habilidades Comunicativas. A estos últimos se les solicitó los últimos 20 minutos del curso para aplicar el instrumento.

La participación fue voluntaria y no implicó retribuciones económicas ni tuvo una incidencia en las calificaciones en el curso. A cada participante se entregó una ficha codificada con tres (3) preguntas sobre datos generales (edad, sexo y carrera) y una pregunta de tipo abierta en la que se le solicita definir con sus palabras el término plagio. Una vez obtenida la información, se transcribieron las respuestas a formato de texto Word y se dio inicio al proceso de edición de la información, respetando en todo momento las expresiones originales de sus autores. Finalmente, se procedió al procesamiento de datos.

Para la interpretación de la información se recurrió a la técnica del análisis del contenido, con el apoyo del programa informático Nvivo, versión 10, como ayuda para el manejo y organización de los datos.

Cada participante recibió una codificación y un número correlativo: P1, por ejemplo, hace referencia al primer participante; P2, al segundo participante, y así sucesivamente.

\section{Resultados}

Los hallazgos se agruparon en tres categorías. La primera, denominada «Términos recurrentes», agrupó a todos los términos con los que los estudiantes relacionaron la acción de plagiar; la segunda, denominada «Productos susceptibles de plagio», agrupó a los productos académicos y no académicos susceptibles, para 
los estudiantes, de ser plagiados, y la tercera categoría, "Definición propuesta», agrupa las situaciones más comunes en las que se comete este acto. Cada una de estas categorías emergió del análisis de las definiciones brindadas por los participantes. A continuación, se analizan cada una de estas categorías.

\subsection{Términos recurrentes}

El análisis de contenido de las definiciones dio a conocer que, en 108 casos, los estudiantes usan el término copiar como sinónimo de plagio. En este sentido, las definiciones analizadas coinciden en gran porcentaje con la definición brindada por el DLE. Veamos algunos casos:

P1. Copiar respuestas de otro compañero discretamente.

P67. Copia de información o trabajo para beneficio de una persona.

P100. Copiar respuestas o tareas dentro de la Universidad.

P190. Una manera de copiar información que nos sirve ya sea para un examen.

En segundo lugar, de frecuencia aparece el término persona (49 menciones) para hacer referencia a que plagiar es copiar algo de otra persona. Veamos algunos casos:

P6. Copiar a otra persona.

P99. Es escribir la información de otra persona, sin colocarle la fuente o comillas o haciéndola pasar como si fuera tuya.

En tercer lugar, aparece el término examen como referencia a la evaluación escrita. El vocablo aparece en 37 casos. Veamos algunos de estos:

P6. Cuando en un examen te copias del compañero de al lado o sacas una hoja donde están las respuestas de tu examen.

P7. Copiar respuestas durante el examen. 
En tanto, el término información aparece mencionado en 34 definiciones. Veamos algunos casos:

P2. Copia de información o trabajo para beneficio de una persona.

P27. Es cuando se utiliza información sin permiso citada, ya que no pertenece a su autoría. Por lo tanto, cuando una persona saca información de una página web o un libro sin citar al autor, está dejando de lado la ética profesional.

De lo analizado podemos afirmar que si bien existen similitudes entre las definiciones de los estudiantes y la del DLE, se asume por lo observado que existen elementos concomitantes (como examen, persona, información) con que se relaciona el concepto de plagio, que es el hecho de copiar de un compañero parte de lo que este responde en un examen, o la totalidad. El plagio es un acto deshonesto e ilícito, lo cual queda implícito en la totalidad de las definiciones analizadas; aunque de manera explícita, ambos términos solo aparecen — debemos señalarlo- en cuatro definiciones.

\subsection{Productos susceptibles de plagio}

Los productos que señalan los estudiantes como susceptibles de plagio son los exámenes, trabajos escritos y apuntes de clase. Los estudiantes se ubican en el contexto universitario y no salen de este. Persiste la creencia de que los exámenes y trabajos que se dejan en la universidad serán siempre tema de preocupación para los estudiantes. Las acciones que involucran estas actividades someten al alumno a una presión considerable hasta el punto de verse tentado de tomar caminos nada honestos para lograr el éxito. Esta explicación se sustenta en lo manifestado por los participantes. Veamos algunos casos:

P17. Plagio, palabra usada para aquella persona que está copiando algo y presentado como el auténtico autor. Otro puede ser el plagio realizado en un examen de tener algún (sic) porque con todas las respuestas

P20. Copiarme en horas de examen sea por celular o por medio de un compañero. 
P35. Hacer copiar a un compañero algún trabajo o ya sea en un examen. P36. Copiar la respuesta de alguna persona en un examen o trabajo.

Lo dicho por los estudiantes confirma la idea de que plagiar es un acto cuestionable, aunque no ahondan en el alcance de este, es decir, no relacionan el acto de plagiar con verbos como robar. Quizá el término que mayor alcance tiene a lo manifestado por los estudiantes es hacer trampa.

\subsection{Definición propuesta a partir de los hallazgos}

A partir de todos los elementos analizados, se puede elaborar una definición aproximativa de plagio. Para ello, es necesario, primero, agrupar los términos recurrentes manifestados por los participantes y luego añadirle las marcas de verbo transitivo, juvenil y popular, ya que esta definición o idea está dentro de este ambiente. Veamos esa agrupación:

\section{COPIAR + PERSONA + EXAMEN, INFORMACIÓN, TRABAJO $=$ COPIAR A UNA PERSONA O COMPAÑERO INFORMACIÓN DURANTE UN EXAMEN O TRABAJO}

Ahora, veamos la definición propuesta:

plagiar. tr. «juv., pop.». Copiar a una persona o compañero información durante un examen o trabajo.

Como vemos, en la definición construida a partir de lo que piensan los estudiantes encontramos que no hay un cuestionamiento hacia este tipo de prácticas. En segundo plano quedarían términos como deshonesto, ilícito y trampa. Está definición no se centra en cuestionar el plagio como algo legal o ilegal, ni tampoco interesa si su acción conlleva algún beneficio para quien lo comete. La idea que se maneja es la acción simplemente, sin implicancias subjetivas o valoraciones. Ya algunos estudios previos señalaban que el plagio era un acto permitido 
https://doi.org/10.46744/bapl.201901.005

y hasta cierto punto aceptado como algo normal entre los estudiantes universitarios (Ramos et al., 2019).

\section{Conclusiones}

El estudio arroja datos interesantes sobre los términos más frecuentes con que los estudiantes peruanos, específicamente de la universidad privada de Arequipa, entienden el plagio. En general, existe conocimiento básico sobre su significado, ya que se aproxima a la definición dada en el DLE, cuyas entradas, por ser un diccionario de lengua, no son de especialidad, pero sí nos ayudan a comprender las palabras. No obstante, la concepción de plagio como la 'acción de copiar' no conlleva detalles como sus consecuencias o causas en caso se practique. Así mismo, quedan fuera adjetivos como deshonesto o trampa, calificativos con los que usualmente se relaciona al acto de plagiar.

Ahora bien, dada la condición de los participantes (estudiantes ingresantes) y el contexto en el que fue llevado a cabo el estudio, no sorprende que el acto de copiar ocurra entre compañeros (unos copian de otros), en el que los productos académicos más susceptibles de plagio son los exámenes o los trabajos específicos. Pero durante el progreso de su carrera, se espera que el concepto o idea de plagio sea más elaborado y preciso.

Del análisis se puede desprender una definición aproximada, la cual se enfoca en la acción de plagiar, el afectado (autor que es víctima del plagio) y el producto susceptible de ser plagiado, tres elementos que también son asumidos en la definición propuesta por la RAE en su diccionario.

Finalmente, los autores recomiendan a las instituciones difundir no solo lo que es el plagio, sino sus consecuencias y sanciones, con el fin de poder lograr que los estudiantes asuman el compromiso de actuar con honestidad, sobre todo en etapas tan importantes como lo son los primeros años de educación superior. 


\section{BIBLIOGRAFÍA}

BEREITER, M., y SCARDAMALIA, C. (1992). Dos modelos explicativos de los procesos de composición escrita. Revista Infancia y aprendizaje, 58, 43-64.

CASTRO, Y., YOPLAC-LOPEZ, B., CARPIO-TELLO, K., SIHUAYTORRES, K. y COSAR-QUIRÓZ, J. (2016). Percepción del plagio académico en estudiantes de odontología. Educación Médica, 19(3), 141-145.

CEBRIÁN-ROBLES, V., RAPOSO-RIVAS, M., CEBRIÁN DE LA SERNA, M. y SARMIENTO-CAMPOS, J. (2018). Percepción sobre el plagio académico de estudiantes universitarios españoles. Educación XXI, 21(2), 105-129.

ESPINOSA, A., CASTELlaRíN, M., y BIAGIONI, F. (2013). Prácticas Académicas deshonestas en exámenes escritos. Análisis de una muestra de alumnos de $1^{\circ}$ año de la carrera de Psicología de la UNR. En V Congreso Internacional de Investigación y Práctica Profesional en Psicología XX Jornadas de Investigación Noveno Encuentro de Investigadores en Psicología del MERCOSUR. Facultad de Psicología, Universidad de Buenos Aires, Buenos Aires.

HEXMAN, I. (1999). Academic Plagiarism Defined. Departament of Religious Studies University of Calgary. Recuperado de http://www. ucalgary.ca/ hexham/study/ plag.html

IBAÑEZ, E., TAGLIABUE, R. y ZANGARO, M. (2007). Investigar para saber. Saber para escribir. Buenos Aires: Temas.

JORDAN, I. (2001). College Student Cheating: The Role of Motivation, Perceived Norms, Attitudes, and Knowledge of Institutional Policy. Ethics \& Behavior, 11(3.), 233-24. 
https://doi.org/10.46744/bapl.201901.005

LAMBERT, E., HOGAN, N. y BARTON, S. (2003). Collegiate Academic Dishonesty Revisited: What Have They Done, How Often Have They Done It, Who Does It, and Why Did They Do It? Electronic Journal of Sociology, 7(4). Recuperado de http://www.sociology.org/content/vol7.4/ lambert_etal.html

LOPERA, J. D., RAMÍREZ, C. A. ZULUAGA, M. U. y ORTÍZ, J. (2004). El método analítico como método natural. Nómadas. Revista Crítica de Ciencias Sociales y Jurídicas. 25(1). 327-353.

LÓPEZ-GIL, K. y FERNÁNDEZ-LÓPEZ, M. (2019). Representaciones sociales de estudiantes universitarios sobre el plagio en la escritura académica. ÍKALA, 24(1), 119-134.

MCCABE, D. y TREVINO, L. (1993). Academic Dishonesty: Honor Codes and Other Contextual Influences. Journal of Higher Education, 64(5), 522-538.

MOLINA, F., VELÁSQUEZ, J. D., RÍOS, S., CALFUCOY, P. A. y COCIÑA, M. (2011). El fenómeno del plagio en documentos digitales: un análisis de la situación actual en el sistema educacional chileno. Revista Ingeniería de Sistemas, 25, 5-28.

RAMOS, T., DAMIÁN, E., INGA, M., ARIAS, D. y CAURCEL, M. (2019). Actitudes hacia el plagio en estudiantes de Administración de Empresas de dos universidades privadas en Arequipa. Propósitos y Representaciones, 7(1). Doi: http:// dx.doi.org/10.20511/pyr2019.v7n1.264

REBOLLO-QUINTELA, N., ESPIÑEIRA-BELLÓN, E., \& MUÑOZCANTERO, J. (2017). Atribuciones causales en el plagio académico por parte de los estudiantes universitarios. Revista de Estudios e Investigación en Psicología y Educación, 6, A6-193. 
ROJAS, M. (2016). Plagio académico. Revista Colombiana de Anestesiología, 38(4). Recuperado de http://www.scielo.org.co/scielo. php?script $=$ sci_arttext\&pid $=$ S0120-33472010000400010

ROSSELOT, E. et al. (2008). Plagio intelectual. Documento de la comisión de ética de la facultad de medicina de la universidad de Chile. Revista médica de Chile, 136, 653-658.

GIRÓN, S. (2008). Anotaciones sobre el Plagio. Recuperado de http://www. usergioarboleda.edu.co/libro\%20plagio.pdf

SUNEDU/UD N. ${ }^{\circ}$ 033-2016. Resolución del Consejo Directivo [en línea]. Diario Oficial El Peruano, Lima, Perú, 8 de setiembre de 2016 [fecha de consulta: 21 de agosto de 2018]. Recuperado de https://intranet.sunedu.gob.pe/ documentos/directorios/127/resolucion-y-reglamentorenati-completo-033.pdf

SUREDA, J., COMAS, R. y MOREY, M. (2009). Las causas del plagio académico entre el alumnado universitario según el profesorado. Revista iberoamericana de Educación 50, 197220. 